BMJ Open

Diabetes

Research

\& Care

\section{Treatment choice, medication adherence and glycemic efficacy in people with type 2 diabetes: a UK clinical practice database study}

To cite: Gordon J, McEwan P, Idris I, et al. Treatment choice, medication adherence and glycemic efficacy in people with type 2 diabetes: a UK clinical practice database study. BMJ Open Diab Res Care 2018;6:e000512. doi:10.1136/ bmjdrc-2018-000512

- Additional material is published online only. To view please visit the journal online (http://dx.doi.org/10.1136/ bmjdrc-2018-000512).

Received 10 January 2018 Revised 7 March 2018 Accepted 12 April 2018

Check for updates

${ }^{1}$ Health Economics \& Outcomes Research Ltd, Cardiff, UK

${ }^{2}$ School of Medicine, University of Nottingham, Nottingham, UK ${ }^{3}$ Department of Public Health, University of Adelaide, Adelaide, Australia

${ }^{4}$ Swansea Centre for Health Economics, Swansea University, Swansea, UK

${ }^{5}$ Diabetes Resource Centre, Llandough Hospital, Cardiff, UK ${ }^{6} \mathrm{Global}$ Outcomes Research, Takeda Development Centre Europe Ltd, London, UK

Correspondence to Dr Jason Gordon; jason.gordon@heor.co.uk

\section{ABSTRACT}

Objective Using primary care data obtained from the UK Clinical Practice Research Datalink, this retrospective cohort study examined the relationships between medication adherence and clinical outcomes in patients with type 2 diabetes.

Research design and methods Data were extracted for patients treated between 2008 and 2016, and stratified by oral antihyperglycemic agent (OHA) line of therapy (mono, dual or triple therapy). Patients were monitored for up to 365 days; associations between medication possession ratio (MPR) and outcomes at 1 year (glycated hemoglobin A1c ( $\mathrm{HbA1c}$ ), weight and hypoglycemia incidence) were assessed using linear regression modeling and descriptive analyses.

Results In total, 33849 patients were included in the study ( $n=23925$ OHA monotherapy; $n=8406$ OHA dual therapy; $n=1518$ OHA triple therapy). One-year change in $\mathrm{HbA} 1 \mathrm{C}$ was greater among adherent $(-0.90$ to $-1.14 \%$; -9.8 to $-12.5 \mathrm{mmol} / \mathrm{mol}$ ) compared with non-adherent patients ( -0.49 to $-0.69 \% ;-5.4$ to $-7.5 \mathrm{mmol} / \mathrm{mol}$ ). On average, adherent patients had higher hypoglycemia event rates than non-adherent patients (rate ratios of 1.24, 1.10 and 2.06 for $\mathrm{OHA}$ mono, dual and triple therapy cohorts, respectively) and experienced greater weight change from baseline. A 10\% improvement in MPR was associated with $-0.09 \%(-1.0 \mathrm{mmol} / \mathrm{mol}),-0.09 \%(-1.0 \mathrm{mmol} / \mathrm{mol})$ and $-0.21 \%(-2.3 \mathrm{mmol} / \mathrm{mol})$ changes in $\mathrm{HbA} 1 \mathrm{c}$ for $\mathrm{OHA}$ mono, dual and triple therapy cohorts, respectively.

Conclusions For patients with type 2 diabetes, increasing medication adherence can bring about meaningful improvements in $\mathrm{HbA1C}$ control as the requirement for treatment escalation increases. Regimens associated with weight loss and the avoidance of hypoglycemia were generally associated with better medication adherence and improved glycemic control.

\section{INTRODUCTION}

Optimal glycemic management is essential to avoid the downstream health and economic consequences of type 2 diabetes, both at a patient and population level. In addition to dietary and lifestyle factors, choice of medication for type 2 diabetes represents a key determinant of the achievement and maintenance of glycemic control.

\section{Significance of this study}

What is already known about this subject?

- The glucose-lowering efficacy of type 2 diabetes medications may only be realized through optimal patient adherence; a factor negatively impacted by treatment-related side effects such as weight gain and hypoglycemia.

What are the new findings?

- Using real-world data from 33849 patients with type 2 diabetes in UK clinical practice, this study found that across oral antihyperglycemic agent (OHA) lines of therapy (mono, dual or triple therapy) regimens typically associated with weight loss and lower incidence of hypoglycemia were generally associated with better medication adherence, which in turn was related to improved glycemic control.

How might these results change the focus of research or clinical practice?

- For patients with type 2 diabetes requiring therapy escalation across the OHA treatment paradigm, data from this study have identified important determinants of optimal medication adherence and glycemic management.

The evolution of type 2 diabetes is characterized by elevations in glycated hemoglobin A1c (HbA1c) levels over time and an ongoing requirement to adjust medication with respect to glucose-lowering efficacy, patient preferences and side effect profiles. In patients newly diagnosed with type 2 diabetes, metformin (MET) or sulfonylurea (SU) are common first-line therapeutic strategies. ${ }^{1}$ These therapies often elicit a good, initial glucose-lowering response; however, in patients who experience intolerable side effects (including gastrointestinal symptoms and/or hypoglycemia) or do not achieve sufficient glycemic control with these therapies (initially or over time as the disease progresses), there is a requirement 
to escalate to alternative monotherapy or combination regimens. These may include other oral antihyperglycemic agents (OHA) such as thiazolidinedione (TZD), sodium-glucose co-transporter-2 inhibitors (SGLT-2i) and dipeptidyl peptidase-4 inhibitors (DPP-4i) or injectable regimens including glucagon-like peptide-1 (GLP-1) and insulin-based therapies. ${ }^{1}$ These alternative classes of medication are each associated with unique efficacy and side effect profiles which, on their own or in combination, may determine patient adherence and levels of glycemic control. $^{2}$

The glucose-lowering potential of medication may only be realized through optimal medication adherence. Factors recognized by patients with diabetes to negatively impact their adherence to medication include the incidence of treatment-related side effects, particularly weight gain and hypoglycemia. ${ }^{3}$ Research evaluating the impact of administering OHAs intermittently, rather than as recommended, ${ }^{1}$ found that patients with type 2 diabetes and decreasing levels of medication adherence were consistently associated with a smaller HbAlc reductions compared with adherent patients. ${ }^{4}$ In clinical terms, the UK Prospective Diabetes Study demonstrated that each 1\% (11 $\mathrm{mmol} / \mathrm{mol}$ ) reduction in $\mathrm{HbAlc}$ reduced the risk of diabetes-related death by $21 \%$, myocardial infarction by $14 \%$, stroke by $12 \%$ and microvascular complications by $37 \% .^{5}$ In economic terms, Baxter and colleagues reported that by maintaining HbAlc levels recommended by the National Institute for Health and Care Excellence, cost reductions could total £299 million over 5 years across the UK adult type 2 diabetes population, rising to $£ 4.5$ billion over 25 years. ${ }^{6}$ In general, poor medication adherence in type 2 diabetes has been found to negatively influence time to treatment intensification, ${ }^{7}$ rate of hospitalizations ${ }^{8}$ and healthcare expenditure $^{9}$ related to diabetes-related morbidity and mortality.

Given that type 2 diabetes is characterized by the need to adjust treatments to maintain glycemic control over time, and that different treatments can elicit different levels of adherence due to side effect profiles, there is a requirement to establish the inter-relationships between these factors and HbA1c outcomes. Associations between treatment, outcomes and adherence have previously been evaluated ${ }^{4}{ }^{11}$; however, such research has focused on specific classes of therapy or restricted populations, without addressing the evolution of type 2 diabetes and outcomes associated with OHA therapy escalation in a general clinical practice population. In light of this, the present retrospective cohort study aimed to generate evidence, based on a large cohort of patients with type 2 diabetes treated in UK clinical practice, to quantify inter-relationships between OHA treatment choice, line of therapy, medication adherence, HbA1c, weight and hypoglycemia, which may inform clinical management and health policy.
RESEARCH DESIGN AND METHODS

\section{Study design}

This study retrospectively analyzed patients with type 2 diabetes who were prescribed one of three treatment regimens (OHA monotherapy, dual or triple therapy) between 1 January 2008 and 31 December 2016. The index date was defined as the date of treatment initiation, and the baseline data period was defined as the quarter prior to the index date. Data were extracted quarterly, with measurements based on the last available record for each study variable within each period. Patients were monitored for up to 365 days post-index date, or until death, treatment cessation, treatment intensification to a non-examinable regimen, lost to follow-up or the end of the study period.

\section{Data source}

The Clinical Practice Research Datalink (CPRD; formerly the General Practice Research Database) contains primary care data for approximately 11.3 million individuals registered with selected general practitioners in the UK. ${ }^{12}$ In this study, patient-level data were extracted from the CPRD to obtain patient demographic and lifestyle information, as well as information on medical diagnoses, symptoms, referrals, hospitalizations, deaths and prescriptions, for each patient. Prescriptions are generated directly within the system and contain the name of the preparation, instructions for use, route of administration, dose and number of tablets for each entry. Primary care data derived from the CPRD have been validated and demonstrated to be of high quality ${ }^{13} 14$ and used in previous observational research of diabetes, ${ }^{15-17}$ including the assessment of adherence and HbAlc. ${ }^{4}$

\section{Population}

Patients eligible for inclusion in this study were aged $\geq 18$ years at index date with a diagnosis of type 2 diabetes, based on a Read code for type 2 diabetes or a prescription record of OHA. Patients with gestational or type 1 diabetes (based on read codes for type 1 diabetes or a prescription record indicating first-line insulin therapy), diagnosis of polycystic ovary syndrome or malignant disease prior to index date and/or during follow-up were excluded.

For each treatment cohort, eligible patients were required to have at least a 365-day record of prescription coverage and a minimum of two prescriptions filled for the medication(s) comprising a given regimen. OHA monotherapy included all patients prescribed one OHA over the study period; OHA dual therapy included patients with at least two instances of two simultaneously prescribed OHAs with no instances of additional OHA prescriptions; OHA triple therapy included patients with at least two instances of three simultaneously prescribed OHAs with no instances of additional OHA prescriptions. Patients with prior insulin usage or in receipt of regimens including GLP-1 were excluded due to 
inadequate recording of injectable regimens (ie, dose and preparation).

By design, patients may have been included in more than one cohort at different times during the study period, provided they met the eligibility criteria for each cohort. Therapy change post-index was defined as cessation of one or more of the medication(s) comprising each regimen and/or initiation of a new treatment.

\section{Derived variables}

Descriptive analyses were undertaken to compare patient profiles, outcomes and patterns of medication adherence within and across treatment cohorts. Patient outcomes of interest included 1-year HbAlc and weight change from baseline (for patients with 365 days of follow-up data), 1-year hypoglycemic event incidence (total events and rate per 1000 patient-years) and medication adherence.

Consistent with methodological approaches advocated by the International Society for Pharmacoeconomics and Outcomes Research, medication adherence was assessed by calculating the medication possession ratio (MPR). ${ }^{18}$ In this study, MPR was defined as the total number of days of available medication (calculated as the quantity of drug prescribed divided by the daily dose), divided by the length of the analysis period. For dual and triple OHA therapies, overall MPR was calculated as the average MPR of all medications comprising each regimen.

If prescription coverage was $<365$ days for a given regimen (due to therapy change or discontinuation) or periods between prescriptions were $>6$ months apart, the analysis period was shortened to coincide with the expected end date of the last valid prescription. Where dose information was absent for prescriptions within the analysis period, but present for a minimum of three prescriptions, the invalid prescriptions were omitted; and the time between the invalid prescription and the next valid prescription was subtracted from the denominator of the MPR calculation.

Patients with an overall MPR of $\geq 80 \%$ (MPR $\geq 0.80$ ) were considered adherent to treatment; non-adherence was defined as MPR $<0.80$. Consistent with the approach of previous studies, patients with MPR calculated as $>120 \%$ were excluded from the analysis. ${ }^{4}$

\section{Statistical analyses}

For baseline patient and treatment characteristics, descriptive analyses for continuous variables (number of patients, mean, SD, median, minimum and maximum values) and categorical variables (the number and proportions of patients) were reported. Statistical significance of between-group comparisons was estimated using $\chi^{2}$ tests (categorical variables) and analysis of variance (continuous variables) to determine significant differences at the $5 \%$ level of testing.

Multivariate regression analyses were based on a general-specific selection methodology, with covariates excluded at the $5 \%$ level of statistical significance. Overall model fit was determined by appropriate goodness-of-fit statistics, including the $\mathrm{R}^{2}$ statistic, likelihood ratio test and Akaike information criterion and Bayesian information criterion. All analyses were undertaken using R software for statistical computing.

Linear regression modeling was used to determine the association between patient variables, $\mathrm{HbAlc}$ and medication adherence. In patients with 365 days of follow-up data, multivariate regression modeling was used to assess the influence of observed covariates (demographic, clinical and socioeconomic factors, other prescriptions, comorbidities and center effects) on MPR and HbAlc change over 1 year.

\section{RESULTS \\ Patient characteristics}

A total of 159799 patients aged $\geq 18$ years within the CPRD database were identified as having type 2 diabetes between 1 January 2008 and 31 December 2016. After inclusion and exclusion criteria were applied, 33849 patients were considered eligible for this study. Of these, 23925 patients were prescribed OHA monotherapy and met the inclusion criteria for this treatment cohort, while 8406 patients were eligible for inclusion in the OHA dual therapy cohort and 1518 patients were included OHA triple therapy cohort (online supplementary figure S1).

At baseline, patients included in the OHA monotherapy subgroup tended to have lower body weight $\left(94.4 \pm 21.9 \mathrm{~kg}\right.$; body mass index (BMI) $\left.32.9 \pm 6.6 \mathrm{~kg} / \mathrm{m}^{2}\right)$, a shorter duration of diabetes $(0.7 \pm 1.3$ years $)$, lower baseline HbA1c $(7.8 \% \pm 1.6 \% ; 62 \pm 17.5 \mathrm{mmol} / \mathrm{mol})$ and fewer total prescriptions $(19.9 \pm 18.9)$. In contrast, the OHA triple-therapy cohort had increased body weight at baseline $\left(98.2 \pm 21.8 \mathrm{~kg}\right.$; BMI $\left.33.4 \pm 6.6 \mathrm{~kg} / \mathrm{m}^{2}\right)$, a longer duration of diabetes $(3.0 \pm 1.8$ years), higher baseline HbAlc $(9.1 \% \pm 1.5 \% ; 76 \pm 16.4 \mathrm{mmol} / \mathrm{mol})$, greater total prescriptions (26.9 \pm 22.6$)$ and a higher likelihood of receiving lipid-lowering and antihypertensive therapies; potentially reflective of treatment escalation due to the progressive nature of diabetes and comorbid diseases. Other variables including baseline age, proportion of male patients, clinical measurements and type 2 diabetes-related event history were comparable across treatment cohorts. Within each OHA cohort, MET, SU and DPP-4i were the most commonly prescribed regimens (table 1 ).

\section{Patient outcomes}

Treatment with OHA monotherapy was associated with a mean $\mathrm{HbA} 1 \mathrm{c}$ reduction of $-0.8 \% \quad(-8.7 \mathrm{mmol} / \mathrm{mol})$ over 1 year compared with $-0.9 \%(-9.8 \mathrm{mmol} / \mathrm{mol})$ and $-1.0 \%(-10.9 \mathrm{mmol} / \mathrm{mol})$ in patients treated with OHA dual and triple therapy, respectively (table 2). Across treatments and cohorts, patients who were adherent to therapy (MPR $\geq 0.80$ ) generally experienced significantly greater reductions in HbAlc over 1 year $(-0.90$ to $-1.14 \% ;-9.8$ to $-12.5 \mathrm{mmol} / \mathrm{mol}$ ) than those considered non-adherent $(-0.49$ to $-0.69 \% ;-5.4$ to $-7.5 \mathrm{mmol} / \mathrm{mol})$. 
Table 1 Baseline characteristics of patients with type 2 diabetes in each oral antihyperglycemic agent (OHA) therapy cohort

\begin{tabular}{lll} 
OHA therapy cohort & & \\
\hline $\begin{array}{l}\text { OHA monotherapy } \\
(n=23925)\end{array}$ & $\begin{array}{l}\text { OHA dual therapy } \\
(n=8406)\end{array}$ & $\begin{array}{l}\text { OHA triple therapy } \\
(n=1158)\end{array}$
\end{tabular}

\begin{tabular}{|c|c|c|c|}
\hline \multicolumn{4}{|l|}{ Baseline patient characteristics } \\
\hline Age (years), mean (SD) & $59.2(12.9)$ & $56.9(11.9)$ & $56.6(10.6)$ \\
\hline Male sex, n (\%) & $14356(60.0 \%)$ & $5475(65.1 \%)$ & $1061(69.9 \%)$ \\
\hline Current smoker, n (\%) & $2388(10.0 \%)$ & $750(8.9 \%)$ & $123(8.1 \%)$ \\
\hline Duration of diabetes (years), mean (SD) & $0.7(1.3)$ & $1.9(1.8)$ & $3.0(1.8)$ \\
\hline BMI $\left(\mathrm{kg} / \mathrm{m}^{2}\right)$, mean $(\mathrm{SD})$ & $32.9(6.6)$ & $32.8(6.6)$ & $33.4(6.6)$ \\
\hline HbA1c (\%), mean (SD) & $7.8(1.6)$ & $8.6(1.6)$ & $9.1(1.5)$ \\
\hline $\mathrm{HbA1c}(\mathrm{mmol} / \mathrm{mol})$, mean (SD) & $62(17.5)$ & $70(17.5)$ & $76(16.4)$ \\
\hline \multicolumn{4}{|l|}{ Regimens, n (\%) } \\
\hline OHA monotherapy & $23925(100.0 \%)$ & - & - \\
\hline MET & 21628 (90.4\%) & - & - \\
\hline SU & $1758(7.3 \%)$ & - & - \\
\hline DPP-4i & $423(1.8 \%)$ & - & - \\
\hline Other & $116(0.5 \%)$ & - & - \\
\hline OHA dual therapy & - & $8406(100.0 \%)$ & - \\
\hline $\mathrm{MET}+\mathrm{SU}$ & - & $4871(57.9 \%)$ & - \\
\hline MET+DPP-4i & - & $2448(29.1 \%)$ & - \\
\hline $\mathrm{MET}+\mathrm{TZD}$ & - & $466(5.5 \%)$ & - \\
\hline MET+SGLT-2i & - & $232(2.8 \%)$ & - \\
\hline SU+DPP-4i & - & $223(2.7 \%)$ & - \\
\hline Other & - & $166(2.0 \%)$ & - \\
\hline OHA triple therapy & - & - & $1518(100.0 \%)$ \\
\hline MET+SU+DPP-4i & - & - & 985 (64.9\%) \\
\hline $\mathrm{MET}+\mathrm{SU}+\mathrm{TZD}$ & - & - & $210(13.8 \%)$ \\
\hline MET+DPP-4i+SGLT-2i & - & - & $119(7.8 \%)$ \\
\hline MET+DPP-4i+TZD & - & - & $104(6.9 \%)$ \\
\hline MET+SU+SGLT-2i & - & - & $68(4.5 \%)$ \\
\hline Other & - & - & $32(2.1 \%)$ \\
\hline \multicolumn{4}{|l|}{ Prescriptions } \\
\hline Mean (SD) & $19.9(18.9)$ & $22.8(19.4)$ & $26.9(22.6)$ \\
\hline $\begin{array}{l}\text { Patients in receipt of lipid-lowering therapy, } \\
n(\%)\end{array}$ & $17462(73.0 \%)$ & $6512(77.5 \%)$ & 1274 (83.9\%) \\
\hline $\begin{array}{l}\text { Patients in receipt of antihypertensive } \\
\text { therapy, } \mathrm{n}(\%)\end{array}$ & $14460(60.4 \%)$ & 5037 (59.9\%) & $973(64.1 \%)$ \\
\hline \multicolumn{4}{|l|}{ Complications at baseline, $n(\%)$} \\
\hline Microvascular complications* & $429(1.79 \%)$ & $393(4.68 \%)$ & $210(13.83 \%)$ \\
\hline Macrovascular complications $\dagger$ & $932(3.90 \%)$ & $261(3.10 \%)$ & $92(6.06 \%)$ \\
\hline Other complications $\ddagger$ & $88(0.37 \%)$ & $62(0.74 \%)$ & $42(2.77 \%)$ \\
\hline
\end{tabular}

*Microvascular complications include diabetic nephropathy, neuropathy (comprising neuropathy, ulcer and amputation) and retinopathy (comprising retinopathy, blindness and macular edema).

†Macrovascular complications include congestive heart failure, ischemic heart disease, myocardial infarction and stroke.

fOther complications include nausea, gastrointestinal complications, edema, urinary tract infection, acute pancreatitis, fracture, ketoacidosis and hypoglycemia.

BMI, body mass index; DPP-4i, dipeptidyl peptidase-4 inhibitor; HbA1c, glycated hemoglobin A1c; MET, metformin; SGLT-2i, sodiumglucose co-transporter-2 inhibitor; SU, sulfonylurea; TZD, thiazolidinedione. 
Table 2 One-year change in glycated hemoglobin A1c ( $\mathrm{HbA1c})$, weight and hypoglycemia incidence, stratified by oral antihyperglycemic agent (OHA) therapy cohort and medication possession ratio (MPR) category

\begin{tabular}{|c|c|c|c|c|c|}
\hline Regimen & $\mathbf{N}$ & $\begin{array}{l}\text { One-year HbA1c change (\%), } \\
\text { mean }(95 \% \mathrm{Cl})\end{array}$ & $\begin{array}{l}\text { One-year HbA1c change } \\
\text { (mmol/mol), mean }(95 \% \mathrm{Cl})\end{array}$ & $\begin{array}{l}\text { One-year weight change }(\mathrm{kg}) \text {, } \\
\text { mean }(95 \% \mathrm{Cl})\end{array}$ & $\begin{array}{l}\text { Hypoglycemia } \\
\text { event rate, } \\
\text { n (per } 1000 \text { patient- } \\
\text { years) }\end{array}$ \\
\hline MPR<80\% & 4401 & $-0.55(-0.61$ to -0.49$)$ & $-6.0(-6.7$ to -5.4$)$ & $-1.64(-1.94$ to -1.34$)$ & $29(6.6)$ \\
\hline MPR $\geq 80 \%$ & 19524 & $-0.90(-0.93$ to -0.87$)$ & $-9.8(-10.2$ to -9.5$)$ & $-2.65(-2.80$ to -2.50$)$ & $160(8.2)$ \\
\hline $\begin{array}{l}\text { OHA dual } \\
\text { therapy }\end{array}$ & 8406 & $-0.9(-1.0$ to -0.9$)$ & $-9.8(-10.9$ to -9.8$)$ & 0.6 (0.4 to 0.8$)$ & $152(18.1)$ \\
\hline $\mathrm{MPR}<80 \%$ & 1610 & $-0.69(-0.79$ to -0.58$)$ & $-7.5(-8.6$ to -6.3$)$ & $0.31(-0.22$ to 0.83$)$ & $27(16.8)$ \\
\hline MPR $\geq 80 \%$ & 6796 & $-0.97(-1.02$ to -0.92$)$ & $-10.6(-11.1$ to -10.1$)$ & 0.67 (0.46 to 0.88$)$ & $125(18.4)$ \\
\hline Difference $^{*}$ & & $-0.28(-0.40$ to -0.17$) ; p<0.001$ & $-3.1(-4.4$ to -1.9$) ; p<0.001$ & $0.36(-0.20$ to 0.93$) ; p=0.210$ & 98 (RR 1.10; $p=0.757)$ \\
\hline Difference* & & $-0.65(-0.92$ to -0.38$) ; p<0.001$ & $-7.1(-10.1$ to -4.2$) ; \mathrm{p}<0.001$ & 0.24 (0.79 to 1.27$) ; \mathrm{p}=0.642$ & 46 (RR 2.06; $p=0.096$ ) \\
\hline
\end{tabular}

${ }^{*}$ Difference relates to MPR $\geq 80 \%$ versus MPR $<80 \%$.

$\mathrm{RR}$, rate ratio.

On average, OHA monotherapy was associated with weight loss over 1 year $(-2.5 \mathrm{~kg})$; within this cohort, weight reductions were greater among adherent $(-2.65 \mathrm{~kg})$ compared with non-adherent $(-1.64 \mathrm{~kg})$ patients. In the dual and triple OHA cohorts, weight tended to increase, more so for adherent $(+0.50$ to $+0.67 \mathrm{~kg})$ compared with non-adherent $(+0.26$ to $+0.31 \mathrm{~kg})$ patients; however, this overall result included a trend of weight gain for SU and TZD-based regimens, and weight loss for DPP-4i and SGLT-2i-based regimens (table 2; online supplementary Table S1).

Despite having higher baseline HbAlc, the incidence of recorded hypoglycemia events (per 1000 patientyears) was greatest in the OHA triple-therapy cohort (38.2), compared with patients receiving OHA monotherapy and dual-therapy regimens (7.9 and 18.1, respectively). Within the OHA monotherapy cohort, treatment with SU was associated with a higher hypoglycemia event rate (15.4) versus MET and DPP-4i therapies (7.3 and 9.5 , respectively). Similarly, the incidence of hypoglycemia was generally higher among patients who received dual-therapy and triple-therapy regimens containing $\mathrm{SU}$, compared with those containing DPP-4i, TZD and/ or SGLT-2i agents (online supplementary Table S1). Observed event rates were higher among adherent versus non-adherent patients in the OHA monotherapy (8.2 vs 6.6), dual-therapy (18.4 vs 16.8) and triple-therapy (42.4 vs 20.6) cohorts, reflecting an increase in hypoglycemia incidence among adherent patients across therapy lines (table 2).

\section{Levels of adherence and glycemic control}

Medication adherence was highest in the OHA monotherapy cohort (81.6\%), followed by dual-therapy $(80.8 \%)$ and triple-therapy $(80.8 \%)$ cohorts (online supplementary Table S1). Adherence by regimen (MPR $\geq 0.80$; $95 \% \mathrm{CI}$ ) for OHA monotherapy was highest for DPP-4i agents $(89.8 \% ; 87.0 \%$ to $92.7 \%)$. For the two most commonly observed dual OHA therapies, MET+DPP-4i (29\% of patients) was associated with a higher level of adherence $(83.3 \% ; 81.8 \%$ to $84.8 \%)$, compared with MET + SU ( $58 \%$ of patients) that had the lowest adherence $(79.6 \% ; 78.5 \%$ to $80.7 \%)$. In patients treated with triple OHA therapies, medication adherence across regimens was similar.

As shown in figure 1, increasing levels of medication adherence were typically associated with greater 1-year HbAlc reductions across all lines of OHA therapy. In absolute terms, the OHA triple-therapy cohort had the greatest level of HbAlc change $(-0.17$ to $-1.20 \%$; -1.9 to $-13.1 \mathrm{mmol} / \mathrm{mol})$, followed by the dual $(-0.60$ to $-1.04 \%$; -6.6 to $-11.4 \mathrm{mmol} / \mathrm{mol})$ and monotherapy $(-0.49$ to $-0.94 \% ;-5.4$ to $-10.3 \mathrm{mmol} / \mathrm{mol}$ ) cohorts.

\section{Predictors of adherence and glycemic control}

Factors predictive of MPR were evaluated for each OHA cohort in linear regression analyses. Across OHA cohorts, patient age, baseline HbA1c, change in BMI and/or change in total cholesterol were significant variables related to MPR variability (table 3 ). Consistent with observations of patient outcomes, coefficient estimates for change in BMI were negative in the OHA monotherapy cohort, and positive in patients receiving OHA dual therapy. Using a regression model that predicted 1-year change in HbA1c as a function of MPR, it was estimated that a $10 \%$ improvement in MPR, when all other variables were held constant, was associated with a $-0.09 \%$ $(-1.0 \mathrm{mmol} / \mathrm{mol}),-0.09 \%(-1.0 \mathrm{mmol} / \mathrm{mol})$ and $-0.21 \%$ 


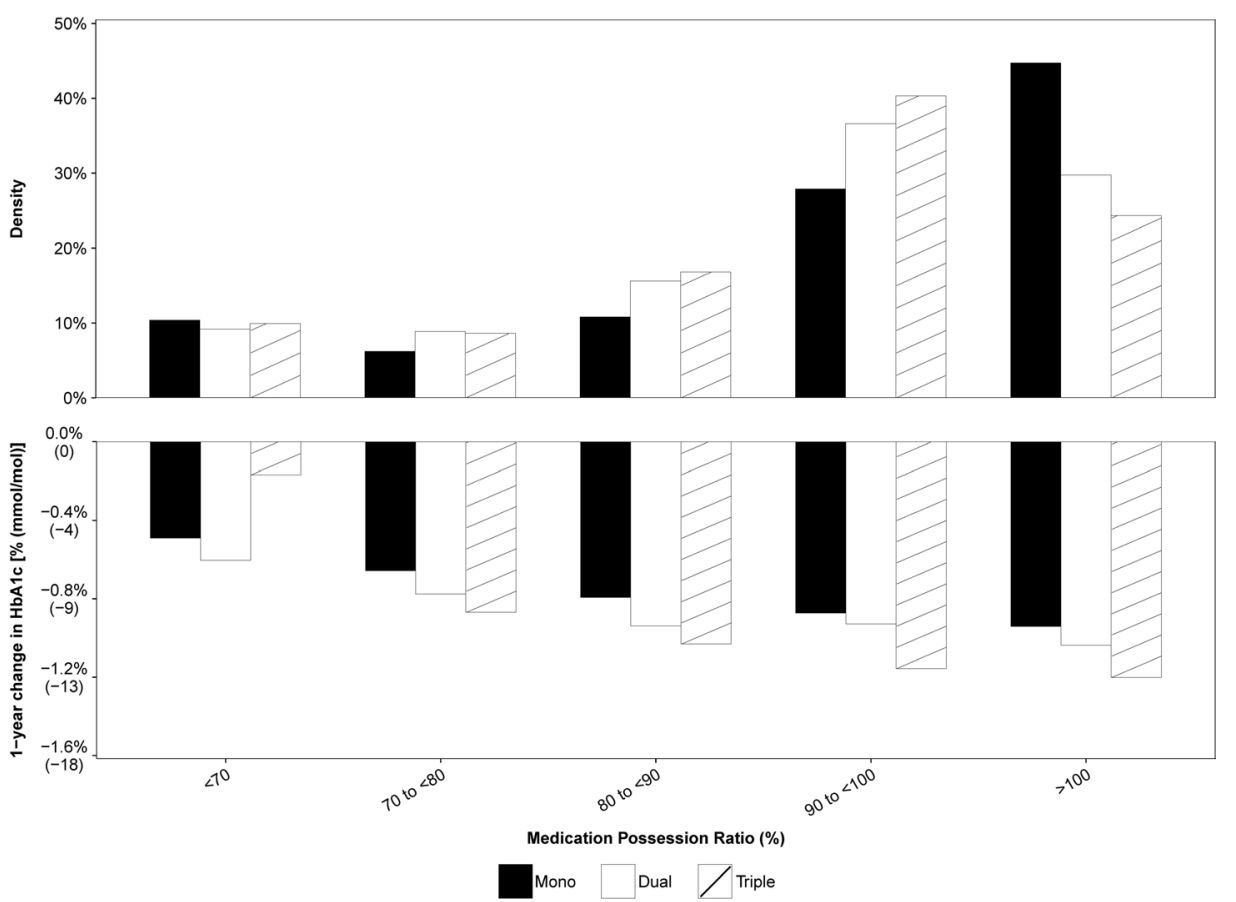

Figure 1 One-year change in glycated hemoglobin $\mathrm{A} 1 \mathrm{c}(\mathrm{HbA} 1 \mathrm{c})$, stratified by medication possession ratio category and oral antihyperglycemic agent therapy cohort.

(-2.3 mmol/mol) change in HbAlc for OHA mono, dual and triple therapy, respectively.

\section{CONCLUSIONS}

Using real-world data from 33849 patients with type 2 diabetes in UK clinical practice, this study sought to evaluate the evolution of type 2 diabetes and outcomes associated with OHA therapy escalation as a function of medication adherence. Analyses found that reduced medication adherence was associated with smaller HbAlc reductions among patients treated with OHA monotherapy, dual-therapy and triple-therapy regimens. One-year reductions in HbAlc were greater among adherent $(-0.90$ to $-1.14 \%$; -9.8 to $-12.5 \mathrm{mmol} / \mathrm{mol})$ compared with non-adherent patients $(-0.49$ to $-0.69 \%$; -5.4 to $-7.5 \mathrm{mmol} / \mathrm{mol}$ ) and a $10 \%$ improvement in MPR was associated with a $-0.09 \%(-1.0 \mathrm{mmol} / \mathrm{mol}),-0.09 \%$ $(-1.0 \mathrm{mmol} / \mathrm{mol})$ and $-0.21 \%(-2.3 \mathrm{mmol} / \mathrm{mol})$ change in HbA1c for OHA monotherapy, dual therapy and triple therapy, respectively. Collectively, these findings support the notion that modest increases in medication adherence can bring about meaningful improvements in $\mathrm{HbA1c}$ control as the requirement to escalate therapy increases.

On average, hypoglycemia was more frequent among adherent patients compared with non-adherent patients, with rate ratios of $1.24,1.10$ and 2.06 associated with OHA monotherapy, dual therapy and triple therapy, respectively. Within each OHA cohort, adherent patients tended to lose more weight (OHA monotherapy) or gain more weight (OHA dual and triple therapy) compared with non-adherent patients. These findings are consistent with a regimen-outcome interaction that may be mediated via medication adherence. For instance, across the OHA treatment paradigm, regimens typically associated with weight loss and lower incidence of hypoglycemia were generally associated with better medication adherence and improved glycemic control.

The health economic value of type 2 diabetes medication is driven by its expected therapeutic profile, in addition to patient and clinician treatment preferences. Key barriers to achieving good medication adherence include treatment-related hypoglycemia and weight gain $^{3}$; in this context, guidelines suggest that DPP-4i or SGLT-2i therapies are associated with weight loss or neutrality and low risk of hypoglycemia, while TZD regimens cause weight gain and SU-based therapies are associated with both weight gain and moderate hypoglycemia risk. ${ }^{19}$ Data arising from this study coincide with such guidance and extend this notion to demonstrate that patients treated with $\mathrm{OHA}$ regimens associated with weight loss and lower hypoglycemia frequency tended to achieve better adherence and HbAlc control. Importantly, this was observed across all lines of OHA therapy, suggesting that adherence may be an increasingly important determinant of glycemic control as patients escalate from OHA monotherapy to dual-therapy and triple-therapy regimens.

To our knowledge, this is the first study to evaluate a large general cohort of UK patients with type 2 diabetes in order to assess the associations between adherence and clinical outcomes across the OHA treatment paradigm. This research builds on existing studies that demonstrate associations between adherence and HbAlc control, ${ }^{4}$ 
Table 3 Multiple linear regression model predicting 1-year glycated hemoglobin A1c $(\mathrm{HbA1c})$ change as a function of medication possession ratio (MPR), stratified by oral antihyperglycemic agent (OHA) therapy cohort

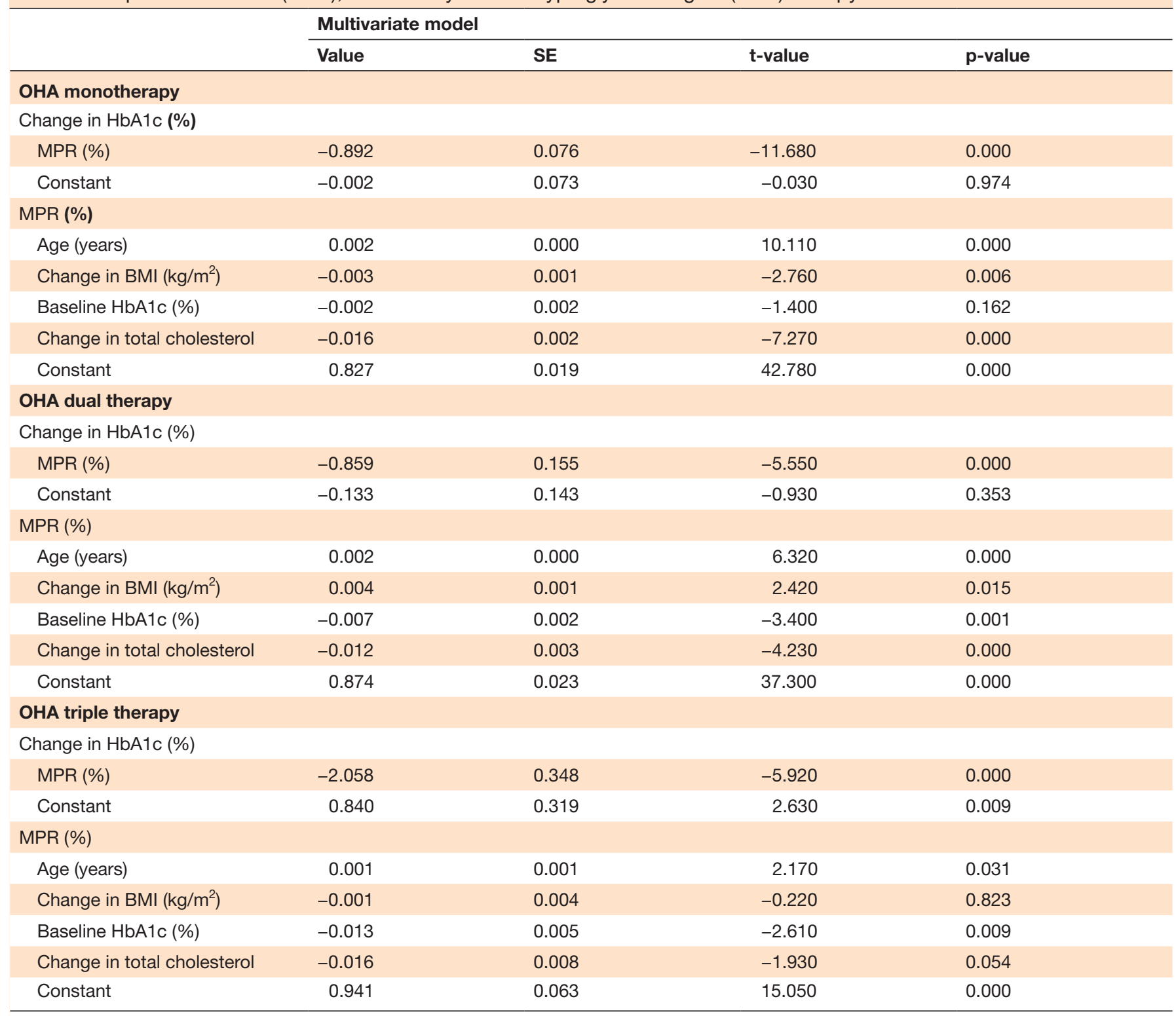

BMI, body mass index.

and hypoglycemia and cardiovascular risk, ${ }^{20}$ in specific patient populations or for individual treatments.

There are several important limitations associated with this research. Observational studies that stratify outcomes by therapy type are potentially subject to the bias of 'confounding by indication', where observed patterns within the data are a function of the patient phenotype, which may in turn dictate the prescription of a specific therapy. Potential confounding factors may not be captured within the CPRD database (such as educational and professional status); thus, predicting causality between treatment and outcome should be interpreted with these limitations in mind. To further minimize the impact of confounding in this study, homogenous OHA cohorts were selected for analysis; and stratification and statistical adjustment were used as the principal methods of accounting for differences in patient type and prescribing choice on study outcomes.

An additional limitation of CPRD-derived data relates to the under-reporting of hypoglycemia, as patients may often self-manage an event and not present to a physician. While there are no data to confirm this, we consequently expect that fewer hypoglycemic events were captured by the CPRD and that such events were more likely to be severe. As a result, data describing hypoglycemia incidence, and its influence on medication adherence and clinical outcomes, may be underestimated in this retrospective study. The under-reporting of hypoglycemia may have additionally led to spurious comparisons between OHA regimens; for example, the relatively high hypoglycemia event rate observed in patients treated with MET monotherapy compared with SU 
(7.3 vs 15.4 events per 1000 patient-years, respectively) may be attributed to the comparatively low number of patients (21628 vs 1758) and recorded events (158 vs 27 ) in the SU monotherapy cohort. Moreover, although the high incidence of hypoglycemia observed in the OHA triple-therapy cohort may reflect greater HbAlc reductions and/or treatment regimens associated with higher hypoglycemia risk, patient numbers are comparatively low in this cohort, and associated data should thus be interpreted with caution. Finally, we were unable to adequately assess medication adherence in patients treated with injectable agents, including GLP-1 receptor agonist and insulin-based therapies, due to a paucity of CPRD data characterizing factors including doses, preparations or the full range of potential treatment-related side effects. Examining the impact of OHA dosing frequency on medication adherence and treatment outcomes was beyond the design and objectives of the present study; however, as dosing frequency may represent an important determinant of patient adherence and subsequent outcomes, this relationship is indicated as an area for future research.

In conclusion, improving HbAlc is the cornerstone of type 2 diabetes therapy, which serves to manage glycemic control and reduce the risks of diabetes-related complications, morbidity and mortality. Improvements in HbAlc are determined by treatment choice and medication adherence; factors which are interrelated and further confounded by patient type and place in the OHA treatment paradigm. This retrospective study of routinely collected UK primary care data sought to evaluate associations between medication adherence and clinical outcomes among patients with type 2 diabetes treated with OHA regimens. Across all lines of OHA therapy examined, lower MPR was associated with smaller HbA1c reductions, suggesting that increased medication adherence can bring about meaningful improvements in HbA1c control as the requirement to escalate therapy increases. Across the OHA treatment paradigm, regimens associated with weight loss and the avoidance of hypoglycemia were generally associated with better medication adherence and improved glycemic control.

Acknowledgements The authors acknowledge the editorial assistance of $\mathrm{Dr}$ Karina Hamilton, of Health Economics and Outcomes Research (HEOR) Ltd.

Contributors JG and PM were involved in the design, analysis, interpretation and writing of the manuscript. II, ME and JP were involved in the design, interpretation and writing of the manuscript. All authors take responsibility for the integrity of the work as a whole and have given final approval to the version to be published. As guarantor and corresponding author, JG takes full responsibility for the work as a whole, including the study design, access to data and the decision to submit and publish the manuscript.

Funding Sponsorship for this study and article processing charges were funded by Takeda Development Centre Europe Ltd. Support for this assistance was funded by HEOR Ltd.

Competing interests JG, PM, II and ME have served as consultants to and received research funding from Takeda Development Centre Europe Ltd in relation to this study. JP is an employee of Takeda Development Centre Europe Ltd. Patient consent Not required.
Ethics approval The CPRD Independent Scientific Advisory Committee approved the use of the CPRD database for the purposes of this study (protocol number 17 025R).

Provenance and peer review Not commissioned; externally peer reviewed. Data sharing statement No additional data are available.

Open Access This is an Open Access article distributed in accordance with the Creative Commons Attribution Non Commercial (CC BY-NC 4.0) license, which permits others to distribute, remix, adapt, build upon this work non-commercially, and license their derivative works on different terms, provided the original work is properly cited and the use is non-commercial. See: http://creativecommons.org/ licenses/by-nc/4.0/

C) Article author(s) (or their employer(s) unless otherwise stated in the text of the article) 2018. All rights reserved. No commercial use is permitted unless otherwise expressly granted.

\section{REFERENCES}

1. National Institute for Health and Care Excellence. Type 2 diabetes in adults: management. NICE guideline [NG28]. 2015 https://www.nice. org.uk/guidance/ng28 (accessed 23 Feb 2017).

2. Nathan DM, Buse JB, Davidson MB, et al. Management of hyperglycemia in type 2 diabetes: A consensus algorithm for the initiation and adjustment of therapy: a consensus statement from the American Diabetes Association and the European Association for the Study of Diabetes. Diabetes Care 2006;29:1963-72.

3. Larkin AT, Hoffman C, Stevens A, et al. Determinants of adherence to diabetes treatment. J Diabetes 2015;7:864-71.

4. Farmer AJ, Rodgers LR, Lonergan M, et al. Adherence to oral glucose-lowering therapies and associations with 1-year $\mathrm{HbA1c}$ : a retrospective cohort analysis in a large primary care database. Diabetes Care 2016;39:258-63.

5. Stratton IM, Adler Al, Neil HA, et al. Association of glycaemia with macrovascular and microvascular complications of type 2 diabetes (UKPDS 35): prospective observational study. BMJ 2000;321:405-12.

6. Baxter M, Hudson R, Mahon J, et al. Estimating the impact of better management of glycaemic control in adults with Type 1 and Type 2 diabetes on the number of clinical complications and the associated financial benefit. Diabet Med 2016;33:1575-81.

7. Grant R, Adams AS, Trinacty CM, et al. Relationship between patient medication adherence and subsequent clinical inertia in type 2 diabetes glycemic management. Diabetes Care 2007;30:807-12.

8. Lau DT, Nau DP. Oral antihyperglycemic medication nonadherence and subsequent hospitalization among individuals with type 2 diabetes. Diabetes Care 2004;27:2149-53.

9. Balkrishnan R, Rajagopalan R, Camacho FT, et al. Predictors of medication adherence and associated health care costs in an older population with type 2 diabetes mellitus: a longitudinal cohort study. Clin Ther 2003;25:2958-71.

10. Grandy S, Fox KM, Hardy E, et al. Association of Weight Loss and Medication Adherence Among Adults With Type 2 Diabetes Mellitus: SHIELD (Study to Help Improve Early evaluation and management of risk factors Leading to Diabetes). Curr Ther Res Clin Exp 2013;75:77-82.

11. Walz L, Pettersson B, Rosenqvist U, et al. Impact of symptomatic hypoglycemia on medication adherence, patient satisfaction with treatment, and glycemic control in patients with type 2 diabetes. Patient Prefer Adherence 2014;8:593-601.

12. Wood L, Martinez C. The General Practice Research Database. Drug Saf 2004;27:871-81.

13. Khan NF, Harrison SE, Rose PW. Validity of diagnostic coding within the General Practice Research Database: a systematic review. $\mathrm{Br} J$ Gen Pract 2010;60:128-36.

14. Herrett E, Thomas SL, Schoonen WM, et al. Validation and validity of diagnoses in the General Practice Research Database: a systematic review. Br J Clin Pharmacol 2010;69:4-14.

15. Meier $\mathrm{C}$, Kraenzlin ME, Bodmer M, et al. Use of thiazolidinediones and fracture risk. Arch Intern Med 2008;168:820-5.

16. Brauchli YB, Jick SS, Curtin F, et al. Association between use of thiazolidinediones or other oral antidiabetics and psoriasis: A population based case-control study. J Am Acad Dermatol 2008;58:421-9.

17. Gordon J, McEwan P, Evans M, et al. Managing glycaemia in older people with type 2 diabetes: A retrospective, primary care-based cohort study, with economic assessment of patient outcomes. Diabetes Obes Metab 2017;19:644-53. 
18. Peterson AM, Nau DP, Cramer JA, et al. A checklist for medication compliance and persistence studies using retrospective databases. Value Health 2007;10:3-12.

19. Inzucchi SE, Bergenstal RM, Buse JB, et al. Management of hyperglycemia in type 2 diabetes, 2015: a patient-centered approach: update to a position statement of the American Diabetes
Association and the European Association for the Study of Diabetes. Diabetes Care 2015;38:140-9.

20. Khunti K, Davies M, Majeed A, et al. Hypoglycemia and risk of cardiovascular disease and all-cause mortality in insulin-treated people with type 1 and type 2 diabetes: a cohort study. Diabetes Care 2015;38:316-22. 